\title{
Invasive pneumococcal disease in hospitalised children from Lima, Peru before and after introduction of the 7-valent conjugated vaccine
}

\section{Original Paper}

Cite this article: Luna-Muschi A et al (2019). Invasive pneumococcal disease in hospitalised children from Lima, Peru before and after introduction of the 7-valent conjugated vaccine. Epidemiology and Infection 147, e91, 1-5. https://doi.org/10.1017/ S0950268819000037

Received: 21 March 2018 Revised: 8 October 2018 Accepted: 26 December 2018

\section{Key words:}

PCV7; Peru; pneumococcal conjugated vaccine; pneumococcal infections; Streptococcus pneumoniae

Author for correspondence:

T. J. Ochoa, E-mail: Theresa.Ochoa@upch.pe; Theresa.J.Ochoa@uth.tmc.edu (c) The Author(s) 2019. This is an Open Access article, distributed under the terms of the Creative Commons Attribution licence (http:// creativecommons.org/licenses/by/4.0/), which permits unrestricted re-use, distribution, and reproduction in any medium, provided the original work is properly cited.

\author{
A. Luna-Muschi ${ }^{1,2}$, F. Castillo-Tokumori ${ }^{1,2}$, M. P. Deza ${ }^{1,2}$, E. H. Mercado ${ }^{1,2}$, \\ M. Egoavil ${ }^{1,2}$, K. Sedano ${ }^{1,2}$, M. E. Castillo ${ }^{1,2,3}$, I. Reyes ${ }^{2,4}$, E. Chaparro ${ }^{1,2,5}$, \\ R. Hernández ${ }^{1,2,5}$, W. Silva ${ }^{2,6}$, O. Del Aguila ${ }^{2,6}$, F. Campos $^{2,7}$, A. Saenz ${ }^{2,8}$ and
}

T. J. Ochoa ${ }^{1,2,9}$

${ }^{1}$ Universidad Peruana Cayetano Heredia, Lima, Peru; ${ }^{2}$ Grupo Peruano de Investigación en Neumococo (GPIN), Lima, Peru; ${ }^{3}$ Instituto Nacional de Salud del Niño, Lima, Peru; ${ }^{4}$ Hospital de Emergencias Pediatricas, Lima, Peru; ${ }^{5}$ Hospital Nacional Cayetano Heredia, Lima, Peru; ${ }^{6}$ Hospital Edgardo Rebagliati, Lima, Peru; ${ }^{7}$ Hospital Nacional Docente Madre Niño San Bartolome, Lima, Peru; ${ }^{8}$ Hospital Daniel A. Carrion, Lima, Peru and ${ }^{9}$ University of Texas Health Science Center at Houston School of Public Health, Houston, Texas, USA

\begin{abstract}
The objective of this study was to determine the serotype distribution and antibiotic resistance of invasive pneumococcal disease (IPD) strains in children from Lima, Peru, before and after the introduction of the 7-valent pneumococcal conjugate vaccine (PCV7), which was introduced in the national immunisation program on 2009. We conducted a prospective, multicentre, passive surveillance IPD study during 2006-2008 and 2009-2011, before and right after the introduction of PCV7 in Peru. The study was performed in 11 hospitals and five private laboratories in Lima, Peru, in patients $<18$ years old, with sterile site cultures yielding Streptococcus pneumoniae. In total 159 S. pneumoniae isolates were recovered. There was a decrease in the incidence of IPD in children $<2$ years old after the introduction of PCV7 $(18.4 / 100000$ vs. $5.1 / 100000, P=0.004)$. Meningitis cases decreased significantly in the second period $(P=0.036)$ as well as the overall case fatality rate $(P=0.025)$, including a decreased case fatality rate of pneumonia $(16.3 \%$ to $0 \%, P=0.04)$. PCV7 serotypes showed a downward trend. Vaccine-preventable serotypes caused $78.9 \%$ of IPD cases, mainly 14 , $6 \mathrm{~B}, 5,19 \mathrm{~F}$ and $23 \mathrm{~F}$. A non-significant increase in erythromycin resistance was reported. Our findings suggest that the introduction of PCV7 led to a significant decrease of IPD in children under 2 years old and in the overall case fatality rate.
\end{abstract}

\section{Introduction}

It is estimated that around half a million children under 5 years of age die every year in the world due to pneumococcal disease, primarily in developing countries [1]. Furthermore, antibiotic-resistant Streptococcus pneumoniae is a clinical and public health problem around the world. In Latin America, penicillin resistance is found in approximately $50 \%$ of isolates, with significant variations among countries [2].

Introduction of pneumococcal conjugate vaccines around the world has led to substantial reduction of invasive pneumococcal disease (IPD) among vaccinated and unvaccinated children due to the decreases in nasopharyngeal colonisation and transmission of vaccine-type pneumococci (herd effect) [3,4]. On 2009, the 7-valent pneumococcal conjugated vaccine (PCV7) was implemented as part of the Peruvian national immunisation program in a $2+1$ dose schedule. PCV10 was introduced in 2011 and PCV13 was introduced in 2015 .

Surveillance of serotypes is required to measure the impact of vaccination. Knowledge of local and regional patterns of serotype distribution and antibiotic resistance is essential for developing effective strategies for vaccination and treatment protocols both for children and adults. The aim of this study was to compare the incidence of IPD, S. pneumoniae serotype distribution and antibiotic resistance patterns before (2006-2008) and after (2009-2011) PCV7 introduction in Lima, Peru.

\section{Material and methods}

\section{Study design}

This study was conducted during 2 periods: May 2006-April 2008, as part of a previously published study [5] and during July 2009-June 2011; each consisting of 2-year prospective, passive, multicentre surveillance studies of IPD in 11 public hospitals and five private clinical 
laboratories in Lima. Cultures were ordered by the attending physicians based on clinical judgment and the hospital's protocol. We followed the same surveillance methodology in both study periods; the pediatrician from each participating hospital, member of our research group, reviewed all positive cultures for S. pneumoniae at the hospital's microbiology laboratory daily. There was no specific sampling, we included all positive cultures from sterile sites during the surveillance period. The study was approved by the Institutional Review Board of Universidad Peruana Cayetano Heredia (Lima, Peru) and by each participating hospital's board.

\section{Case definition}

Invasive pneumococcal disease cases were defined by isolation of S. pneumoniae from normally sterile sites (blood, cerebral spinal fluid (CSF), pleural fluid, joint fluid, or peritoneal fluid) from patients $<18$ years of age, admitted at the participating hospitals. S. pneumoniae isolates from non-sterile (nasopharynx, pharynx, tonsils, or sputum) or unknown sites were excluded. Clinical and epidemiological data were obtained from the medical charts.

The diagnosis of pneumonia, meningitis, sepsis and other diagnoses were taken from medical records. Pneumococcal pneumonia was defined by a positive blood or pleural fluid culture in the presence of an infectious process with fever and respiratory distress and evidence of pulmonary infiltrates on the chest $\mathrm{X}$-ray. Pneumococcal meningitis was defined by isolation of $S$. pneumoniae in CSF or a positive blood culture in the presence of signs and symptoms of neurological involvement. Pneumococcal sepsis was defined by a positive blood culture in the presence of a systemic inflammatory response syndrome (SIRS).

\section{Incidence rate determination}

We determined the populations served by each hospital in each district of the Province of Lima using an estimate based on each district's population and the number of hospitals and clinics available. It was estimated that $75 \%$ of the total pediatric population is served by the study's participating hospitals and clinics. The mean annual IPD rate (number of cases/100 000 children) was calculated based on rates in the study's two periods among children $<2$ years of age ( 1 month-24 months of age) and $<5$ years of age (1 month-60 months of age). The population of Lima was obtained from the National Institute of Statistics and Informatics (INEI). During the first study period, the city of Lima had a mean population of 249799 children $<24$ months of age and 773280 children $<60$ months of age and during the second study period, a population of 316778 and 791331.5 , respectively.

\section{Laboratory studies}

We transported S. pneumoniae isolates in the blood agar plate, from each hospital to a central laboratory on the day of isolation. $S$. pneumoniae identification was confirmed by conventional microbiology methods based on the colony morphology, alpha hemolysis, Gram stain, bile solubility and optochin susceptibility. We used Etest ${ }^{\oplus}$ (AB Biodisk, Solna, Sweden) to determine antimicrobial susceptibility by minimal inhibitory concentration (MIC) to four antibiotics: ceftriaxone, chloramphenicol, erythromycin and penicillin. Susceptibility was assessed according to the
2017 performance standards of the Clinical and Laboratory Standards Institute (CLSI) [6]. Serotyping was performed by Quellung reaction at the Center for Disease Control and Prevention (CDC), Atlanta, USA.

\section{Statistical analysis}

The data are presented as number of cases and percentages. We compared continuous variables between two sub-groups with $t$ tests if normally distributed and with Wilcoxon rank-sum tests if not normally distributed and compared dichotomous or categorical values between two subgroups with $\chi^{2}$ tests. When comparing small values, we used Fisher's exact test, to approximate a better estimate.

\section{Results}

A total of 159 S. pneumoniae isolates were recovered during both study periods, 101 from 2006 to 2008 and 58 from 2009 to 2011. Patients' age ranged from 1 month to 15.4 years with a median of 14 months in the first period and 29 months in the second period. The main diagnoses in both periods were pneumonia and meningitis (Table 1). Pneumococci were isolated mainly from blood and cerebrospinal fluid. The percentage of meningoencephalitis cases significantly decreased from $38.6 \%$ in the first period to $22.4 \%$ in the second $(P=0.036)$.

Children under 2 years of age are the group with the highest risk for IPD. [5]. The incidence rate of IPD in children $<2$ years of age in Lima was 18.4/1 00000 (17.1/1 00000 in the first year and 19.8/100 000 in the second year) during the first period, showing a significant decrease during the second period, where IPD incidence was only 5.1/100000 (7.2/100000 in the first year and 2.9/1 00000 in the second year $(P=0.004))$. There was also a decrease in the incidence rate in children $<5$ years of age, although not significant. The cumulative cases of IPD before and right after PCV-7 introduction are shown in Figure 1.

The overall case fatality rate showed a significant decrease from $22.0 \%$ to $7.5 \%$ after vaccine introduction $(P=0.025)$. The fatality rate in children $<2$ years was $23.2 \%(16 / 52)$ and $13 \%(3 / 23)$ during the first and second period, respectively. The case fatality rate of pneumococcal pneumonia decreased significantly after vaccine introduction, $P=0.04$. (Table 1).

The comparison of serotype distribution in 2006-2008 against 2009-2011 is summarised in Table 2. PCV7 serotypes represented $67.5 \%$ of isolates during the first period and $56.9 \%$ during the second period; the decrease was not significant. However, nonvaccine serotypes have shown a rise since PCV7 introduction, as they increased from $32.5 \%$ to $43.1 \%$ of IPD strains. When assessing for serotypes included in PCV13, we found that $78.9 \%$ of IPD cases would have been caused by vaccine-preventable serotypes. The most frequently isolated serotypes were serotypes 14, $6 \mathrm{~B}, 5,19 \mathrm{~F}, 23 \mathrm{~F}$ and 19A. During the second period, seven patients received at least one dose of PCV7 and two received PPV23.

All 157 S. pneumoniae isolates were evaluated for antibiotic susceptibility (Table 3). Antibiotic resistance was high for penicillin in both periods; the highest resistance rates were among isolates recovered from cerebrospinal fluid $(46.2 \%$ and $50 \%$, respectively). We found a non-significant increase in resistance to erythromycin $(24.8 \%$ vs. $37.5 \%)$. The most frequently resistant serotypes in were $14(17 / 157), 19 \mathrm{~F}(17 / 157)$ and $6 \mathrm{~B}$ $(12 / 157)$. 
Table 1. General characteristics of children with invasive pneumococcal disease before and after PCV introduction

\begin{tabular}{|c|c|c|}
\hline & $\begin{array}{c}2006-2008 \\
n=101\end{array}$ & $\begin{array}{c}2009-2011 \\
n=58\end{array}$ \\
\hline Age, mean (IQR) & $1.17(0.75-2.88)$ & $2.5(1.17-7.08)$ \\
\hline$<2$ years, $n(\%)$ & $69(68.3)$ & $24(41.4)^{\star}$ \\
\hline 2-6 years, $n(\%)$ & $19(18.8)$ & $14(24.1)$ \\
\hline $6-18$ years, $n(\%)$ & $13(12.9)$ & $20(34.5)^{\star}$ \\
\hline Male sex, $n(\%)$ & $59(48.4)$ & $30(51.7)$ \\
\hline \multicolumn{3}{|c|}{$\begin{array}{l}\text { Source of S. pneumoniae culture, } \\
n(\%)\end{array}$} \\
\hline Blood & $59(58.4)$ & $37(63.8)$ \\
\hline Cerebrospinal fluid & $29(38.7)$ & $11(19.0)$ \\
\hline Pleural fluid & $7(6.9)$ & $5(8.6)$ \\
\hline Other & $6(5.9)$ & $5(8.6)$ \\
\hline \multicolumn{3}{|c|}{ IPD medical condition, $n(\%)$} \\
\hline Pneumonia & $48(47.5)$ & $30(51.7)$ \\
\hline Meningoencephalitis & $39(38.6)$ & $13(22.4)^{\star \star}$ \\
\hline Sepsis-bacteremia & $8(7.9)$ & $8(13.8)$ \\
\hline Other & $3(3.0)$ & $7(12.1)$ \\
\hline Fatal cases, $n / N(\%)$ & $20 / 91(22.0)$ & $4 / 53(7.0)^{\star \star}$ \\
\hline Pneumonia & $7 / 43(16.3)$ & $0 / 29(0.0)^{\star \star}$ \\
\hline Meningitis & $11 / 34(32.3)$ & $2 / 11(18.2)$ \\
\hline Other & $2 / 8(25)$ & $2 / 13(15.4)$ \\
\hline
\end{tabular}

IQR, interquartile range.

${ }^{\star} P<0.001,{ }^{\star \star} P<0.05$.

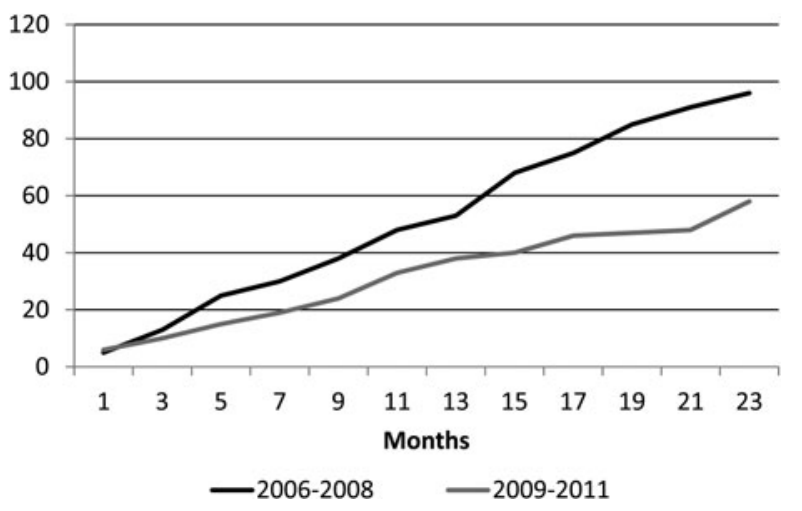

Fig. 1. Cumulative cases of invasive pneumococcal disease (IPD) according to study period. Black line: 2006-2008; grey line 2009-2011.

\section{Discussion}

In this prospective multicenter passive surveillance study in Peru, we found a significant decrease in IPD incidence in the postvaccination period, especially among children $<2$ years of age, comparable with trends found in similar studies performed in Brazil and Uruguay $[7,8]$. This decrease could be due to vaccine effect or a series of other factors that we may have not explored; such as, changes in population socio-economic status and
Table 2. Serotype distribution of Streptococcus pneumoniae before and after $\mathrm{PCV}$ introduction

\begin{tabular}{|c|c|c|}
\hline & $2006-2008$ & $2009-2011$ \\
\hline & $N=99$ & $N=58$ \\
\hline Serotypes & $n(\%)$ & $n(\%)$ \\
\hline 14 & $26(26.3)$ & $11(19.0)$ \\
\hline $6 B$ & $20(20.2)$ & $6(10.3)$ \\
\hline $19 \mathrm{~F}$ & 11 (11.1) & $9(15.5)$ \\
\hline $23 \mathrm{~F}$ & $6(6.1)$ & $6(10.3)$ \\
\hline 4 & $1(1.0)$ & $0(0.0)$ \\
\hline $18 \mathrm{C}$ & $1(1.0)$ & $1(1.7)$ \\
\hline $9 \mathrm{~V}$ & $1(1.0)$ & $1(1.7)$ \\
\hline $7 F$ & $0(0.0)$ & $0(0.0)$ \\
\hline 5 & $6(6.1)$ & $2(3.4)$ \\
\hline 1 & $0(0.0)$ & $2(3.4)$ \\
\hline $19 \mathrm{~A}$ & $4(4.0)$ & $5(8.6)$ \\
\hline 3 & $1(1.0)$ & $2(5.2)$ \\
\hline $6 \mathrm{~A}$ & $4(4.0)$ & $0(0.0)$ \\
\hline Other & $18(18.2)^{a}$ & $12(20.7)^{b}$ \\
\hline \multicolumn{3}{|c|}{ Vaccine serotypes } \\
\hline PCV7 & $66(67.5)$ & $33(56.9)$ \\
\hline PCV10 & $72(72.7)$ & $37(63.8)$ \\
\hline PCV13 & $81(81.8)$ & $44(75.9)$ \\
\hline
\end{tabular}

${ }^{a}$ Other serotypes: Non-typable (three strains); 11A, 15A, 25 (2 strains each), 9N, 12F, 13, 16F, 23A, 34, 38 (one strain each).

b Other serotypes: 10A (four strains); 7C,11A, 12F, 23A, 23B, 34, 35F, 38 (one strain each).

healthcare access. On the other hand, limited laboratory resources could have also been an influencing factor. However, even though the incidence of PCV7 serotypes showed a downward trend, the decrease was not significant, opposing to what was found in the previously mentioned studies. This could be due to an unachieved herd effect, secondary to a vaccination rate of $45 \%$ reported in Peru during the first years of PCV7 introduction [9]. The most common serotypes were $14,6 \mathrm{~B}, 19 \mathrm{~F}$ and $23 \mathrm{~F}$, similar to other countries in the region [10-12]. A previous study made by Hawkins et al. described serotypes and common PBP mutations by whole genome sequencing [13]. They identified the expansion of serotype 19F after PCV7 introduction; specifically, the 19F/ ST1421 lineage, which predicted beta lactam resistance. The current study complements their findings, as it is focused in the epidemiological characteristics of children with IPD and the evaluation of resistance phenotype to other commonly used antibiotics. Almost $80 \%$ of IPD cases were caused by vaccinepreventable serotypes (PCV13). Therefore, higher vaccination coverage should lead to a significant reduction in the burden of disease in the following years.

In developing countries, non-bacteremic pneumonia causes most pneumococcal deaths in children [14, 15]. After vaccine introduction, there were no reported cases of death in children with a diagnosis of pneumonia compared with a case fatality of $14.6 \%$ in the pre-vaccination period. The case-fatality rate was similar in the second period to that of other Latin American countries such as Chile and Brazil $[16,17]$. Even though there 
Table 3. Antimicrobial resistance of Streptococcus pneumoniae strains by minimal inhibitory concentration (MIC)

\begin{tabular}{|c|c|c|c|c|c|c|}
\hline & \multicolumn{3}{|c|}{$2006-2008(n=101)$} & \multicolumn{3}{|c|}{$2009-2011(n=56)$} \\
\hline & Susceptible & Intermediate & Resistant & Susceptible & Intermediate & Resistant \\
\hline & $N(\%)$ & $N(\%)$ & $N(\%)$ & $N(\%)$ & $N(\%)$ & $N(\%)$ \\
\hline \multicolumn{7}{|l|}{ Penicillin $^{a}$} \\
\hline Meningitis & $21(53.8)$ & - & $18(46.2)$ & $7(50.0)$ & - & $7(50.0)$ \\
\hline Non-meningitis & 57 (91.9) & $1(1.6)$ & $4(6.5)$ & $42(100.0)$ & $0(0.0)$ & $0(0.0)$ \\
\hline \multicolumn{7}{|l|}{ Ceftriaxone $^{a}$} \\
\hline Meningitis & $30(76.9)$ & $8(20.5)$ & $1(2.6)$ & $10(71.4)$ & $4(28.6)$ & $0(0.0)$ \\
\hline Non-meningitis & $59(95.2)$ & $1(1.6)$ & $2(3.2)$ & $38(90.5)$ & $2(4.7)$ & $2(4.7)$ \\
\hline Chloramphenicol $^{\mathrm{b}}$ & $89(88.1)$ & - & $12(11.9)$ & $49(89.1)$ & - & $6(10.9)$ \\
\hline Erythromycin & $76(75.2)$ & $0(0.0)$ & $25(24.8)$ & $34(60.7)$ & $1(1.8)$ & $21(37.5)$ \\
\hline
\end{tabular}

an the first period 39 meningitis strains and 62 non-meningitis strains were analysed and in the second period 14 meningitis strains and 42 non-meningitis strains.

$\mathrm{b}$ In the second period 55 strains were analysed for chloramphenicol resistance.

is a clear trend to decreased fatality, several factors, such as inadequate disease registration and suboptimal microbiological identification could have affected pneumococcal strains isolation and lack of PCV7 serotypes decline.

The increasing rate of penicillin resistance of $S$. pneumoniae in Latin America over the past years has raised concerns due to the risk of treatment failure and high healthcare costs [18]. Countries like Mexico and Venezuela have reported resistance rates over $80 \%$ [2]. Our results showed an elevated penicillin-resistance rate $(46.2 \%$ and $50 \%)$ in meningeal strains and showed a nonsignificant decrease in resistance in non-meningeal strains after vaccine introduction. Continuous surveillance is needed in order to guide appropriate empiric treatment of more severe pneumococcal diseases, such as pediatric meningitis.

We found a non-significant increase in erythromycin resistance. Even though this is non-conclusive, we consider it shows an important trend that needs to be followed. For the last 30 years there has been a worldwide increase in S. pneumoniae resistance to macrolides [19-22]. This could render them ineffective in pneumococcal disease as recent studies suggest there is an increased risk of treatment failure of macrolides in respiratory tract infections [22].

The present study has some limitations. First, the data were collected from a passive surveillance study, which could underestimate the incidence of IPD, nevertheless is the starting point for future and more comprehensive studies. In order to have a larger sample size, we would have extended the study period or increase the number of sites. Second, we have not searched for IPD cases in other hospitals beyond the ones included in our study; which also contributes to incidence underestimation. Finally, the results must be considered preliminary, due to the short time after introduction of the vaccine and the low vaccination rates achieved in our country.

\section{Conclusions}

The introduction of the PCV7 in Peru has led to a significant decrease of IPD in children $<2$ years old, with a reduction of PCV7 serotypes, although not significant. Ongoing surveillance of patients with IPD is needed to determine if the decrease in the incidence persists and expands to older populations and to establish the effect of vaccination on serotype distribution and antimicrobial resistance. We intend to conduct follow-up surveillance studies in order to evaluate the effects of PCV10 and PCV13.

\section{Author ORCIDs. (DD T. J. Ochoa, 0000-0002-3227-3906}

Acknowledgements. The authors would like to thank all study personnel, especially the hospital laboratory technicians that isolated the pneumococcal strains. This study was supported by a research grant from Pfizer Laboratories to Grupo Peruano de Investigación en Neumococo (GPIN, Peruvian research group on pneumococcus).

Author contributions. ALM, FCT, MPD, EHM and TJO were responsible for analysis and interpretation of data and drafting of the manuscript. ME, KS, MEC, IR, EC, RH, WS, ODA, FC, AS and TJO were responsible for enrolment, acquisition of samples and data gathering. MEC, IR, EC, RH, WS, ODA, FC, AS and TJO provided expertise in the study design and acquired research funding. All authors reviewed and revised the manuscript critically for important intellectual content.

Conflict of interest. None declared.

\section{References}

1. O'Brien KL et al. (2009) Burden of disease caused by Streptococcus pneumoniae in children younger than 5 years: global estimates. The Lancet $\mathbf{3 7 4}$, 893-902.

2. Jones RN et al. (2013) Susceptibility rates in Latin American nations: report from a regional resistance surveillance program (2011). The Brazilian Journal of Infectious Diseases 17, 672-681.

3. Whitney CG et al. (2003) Decline in invasive pneumococcal disease after the introduction of protein-polysaccharide conjugate vaccine. New England Journal of Medicine 348, 1737-1746.

4. Hicks LA et al. (2007) Incidence of pneumococcal disease due to nonpneumococcal conjugate vaccine (PCV7) serotypes in the United States during the era of widespread PCV7 vaccination, 1998-2004. The Journal of Infectious Diseases 196, 1346-1354.

5. Ochoa TJ et al. (2010) Invasive pneumococcal diseases among hospitalized children in Lima, Peru. Revista Panamericana de Salud Pública 28, 121-127.

6. CLSI (2017) Performance Standards for Antimicrobial Susceptibility Testing, 27th Edn. CLSI supplement M100. Wayne, PA: Clinical and Laboratory Standards Institute.

7. Andrade AL et al. (2016) Evaluating the impact of PCV-10 on invasive pneumococcal disease in Brazil: a time-series analysis. Human Vaccines \& Immunotherapeutics 12, 285-292. 
8. Gabarrot GG et al. (2014) Effect of pneumococcal conjugate vaccination in Uruguay, a middle-income country. PLoS ONE 9, e112337.

9. MEF: Tercer informe de Intervención Pública Evaluada: Servicio de Vacunación Ministerio de Salud, Junio (2011) Available at https:// www.mef.gob.pe/contenidos/presu_publ/ppr/eval_indep/2010_informe_ final_VACUNACIONES.pdf. (Accessed 10 December 2016).

10. dos Santos SR et al. (2013) Serotype distribution of Streptococcus pneumoniae isolated from patients with invasive pneumococcal disease in Brazil before and after ten-pneumococcal conjugate vaccine implementation. Vaccine 31, 6150-6154.

11. Parra EL et al. (2013) Changes in Streptococcus pneumoniae serotype distribution in invasive disease and nasopharyngeal carriage after the heptavalent pneumococcal conjugate vaccine introduction in Bogota, Colombia. Vaccine 31, 4033-4038.

12. Villaseñor-Sierra A et al. (2008) Serotypes and susceptibility of Streptococcus pneumoniae strains isolated from children in Mexico. salud pública de méxico 50, 330-333.

13. Hawkins $\mathbf{P}$ et al. (2017) Key features of invasive pneumococcal isolates recovered in Lima, Peru determined through whole genome sequencing. International Journal of Medical Microbiology. 307, 415-421.

14. World Health Organization (2007) Pneumococcal conjugate vaccine for childhood immunization-WHO position paper. The Weekly Epidemiological Record. 82, 93-104.
15. Williams BG et al. (2002) Estimates of world-wide distribution of child deaths from acute respiratory infections. The Lancet Infectious Diseases 2, 25-32.

16. Fernández JP et al. (2015) Incidencia de egresos por neumonía en niños menores de 24 meses antes y después de la implementación de la vacuna conjugada antineumocócica 10-valente en el Programa Nacional de Inmunizaciones de Chile. Revista chilena de pediatría 86, 168-172.

17. Valenzuela MT et al. (2009) The burden of pneumococcal disease among Latin American and Caribbean children: review of the evidence. Revista Panamericana de Salud Pública 25, 270-279.

18. Valenzuela MT and de Quadros C (2009) Antibiotic resistance in Latin America: a cause for alarm. Vaccine 27, C25-C28.

19. Karcic E et al. (2015) Antimicrobial susceptibility/resistance of Streptococcus pneumoniae. Materia Socio-Medica 27, 180.

20. Linares J et al. (2010) Changes in antimicrobial resistance, serotypes and genotypes in Streptococcus pneumoniae over a 30-year period. Clinical Microbiology and Infection 16, 402-410.

21. Klugman KP (1990) Pneumococcal resistance to antibiotics. Clinical Microbiology Reviews 3, 171-196.

22. McGeer D, Green AK and Low DE, Toronto Invasive Bacterial Diseases Network (2006) Macrolide resistance in bacteremic pneumococcal disease: implications for patient management. Clinical Infectious Diseases 43, 432-438. 